\title{
An Iterative Learning Scheme-Based Fault Estimator Design for Nonlinear Systems with Randomly Occurring Parameter Uncertainties
}

\author{
He Jun (iD, ${ }^{1,2}$ Wei Shanbi $\mathbb{D}^{1,2}$ and Chai Yi ${ }^{1,2}$ \\ ${ }^{1}$ State Key Laboratory of Power Transmission Equipment and System Security and New Technology, Chongqing University, \\ Chongqing 400044, China \\ ${ }^{2}$ College of Automation, Chongqing University, Chongqing 400044, China
}

Correspondence should be addressed to Wei Shanbi; weishanbi@cqu.edu.cn

Received 17 March 2018; Accepted 10 September 2018; Published 1 November 2018

Academic Editor: Michele Scarpiniti

Copyright (C) 2018 He Jun et al. This is an open access article distributed under the Creative Commons Attribution License, which permits unrestricted use, distribution, and reproduction in any medium, provided the original work is properly cited.

\begin{abstract}
This paper deals with fault estimation problem for a class of nonlinear system with parameter uncertainties subjecting to Bernoullidistributed white sequences with known conditional probabilities. In order to reflect the reality more closely, parameter uncertainties are considered in both the state parameter matrix and the output parameter matrix. Compared with existing observer-based fault estimation approaches, the proposed iterative learning observer considers the state error information and fault estimating information from the previous iteration to improve the fault estimation performance in the current iteration. Simultaneously, the stability and convergence of the designed observer are achieved by employing the Lyapunov stability theory. On the other hand, a novel optimal function using expectation is presented to ensure the uniform convergence of the fault estimation scheme, thus reducing the impact of randomly occurring parameter uncertainties. Finally, linear matrix inequality (LMI) is employed to obtain the solutions of sufficient condition for further improvement of iterative learning law performance. The results are suitable for the systems with time-varying uncertainties as well as constant uncertainties. Additionally, a numerical example is given to demonstrate the effectiveness of the proposed design scheme.
\end{abstract}

\section{Introduction}

With the ever-increasing demand on reliability, safety, and maintainability, researches on fault diagnosis [1-4] and fault-tolerant control [5-7] have received more attention in both academic and industrial areas. Fault estimation $[8,9]$ provides the precise magnitude and shape of the faults to guarantee a high system performance, thereby becoming the most critical factor and one of the basic researches in this field. In fault diagnosis problems, fault estimation often helps to generate a residual by comparing measured output with estimated output. By analyzing this residual signal, a decision is made to give a conclusion on whether a fault condition occurred and an attempt is made to determine its location. On the other hand, the fault estimate is usually added into the controller to compensate for the actual fault in faulttolerant control strategies. Hence, fault estimation is further needed for the purpose of active fault-tolerant control to maintain the normal performance of systems.

Up to date, considerable research results on this topic have been reported in the literature; see [10-12] and references therein $[13,14,15,16]$. However, most of the industrial systems are repeated systems [17, 18], and the learning experience and performance from the previous iteration are ignored in conventional fault estimation methods above. With the development of information processing technology, tremendous research efforts have been devoted to design and analysis of a fault estimation scheme by using computer-based learning techniques including neural network-based methods [19-21] and iterative learning scheme-based approaches [22, 23]. In general, neural network-based methods have been capable for complex systems that the model process is unavailable. Nevertheless, for fault estimation problems based on an accurate system 
model, an iterative learning scheme is a more sustainable trajectory. A fault tracking approximator (FTA) and an iterative learning algorithm are utilized to obtain estimates of the fault functions for time-delay systems in [23]. In [24], an iterative learning observer is constructed by using previous output estimation errors and inputs for the purpose of periodically occurring fault estimations in nonlinear timevarying systems. Motivated by predictive and iterative learning control theories, the fault tracking approximator uses iterative algorithms to detect and identify nonlinear system faults, even in the presence of model uncertainty [25]. The latest work of the iterative learning schemebased fault estimation observer is designed for multiphase batch processes with delays, disturbances, and actuator faults [26, 27]; a class of differential time-delay batch processes with actuator faults [28, 29]; and nonlinear systems with randomly changed trial length, period intermittent fault, and time delay [30-32]. Unfortunately, to the best of the authors' knowledge, the iterative learning schemebased fault estimation problem has not been fully investigated, not to mention the case where the systems also involve parameter uncertainties.

In reality, parameter uncertainties usually enter systems in an unknown way and such variations are unknown but with known bounds due to simplified modeling, everchanging environments, and accidental operation. It may reduce systematic performance badly and even cause disaster, which gives the economy and social aspects a huge negative impact. Therefore, many researchers have devoted efforts to fault estimation problems for systems with parameter uncertainties [33-35]. In [33], an auxiliary system is constructed with a certain indefinite quadratic form to deal with the uncertainties in linear discrete time-varying systems with known inputs. In [34], a fault detection and identification procedure is introduced to estimate the fault magnitude, and a fault-tolerant control scheme is presented for linear parameter varying systems. The paper [35] considers robust filtering for discrete uncertain systems where parameter uncertainties are caused by missing measurements, and the measurement missing rate of each sensor is allowed to vary in a range. It should be pointed out that, in the literature mentioned earlier, most results are capable of handling certain uncertainties and may introduce significant conservativeness. Simultaneously, recent works have demonstrated that parameter uncertainties will be randomly occurring in actual systems [36, 37]. It is, therefore, the main purpose of this paper to consider the randomly occurring uncertainties in fault estimation problems for a class of nonlinear systems.

Motivated by these considerations, this paper presents an iterative learning scheme-based fault estimation design for nonlinear systems with randomly occurring parameter uncertainties. Two sets of Bernoulli-distributed white sequences with known conditional probabilities are introduced to describe the parameter uncertainties within a unified framework. Then, an iterative learning observer is designed to estimate the exact information of fault. By employing the Lyapunov stability theory, optimal function is further proposed to ensure the uniform convergence of the error system. Compared with the existing results, the main contributions of this technical note are highlighted as follows:

(1) The existing observer-based fault estimation approaches including sliding mode observer, adaptive observer, and other observers therein are designed by using only the state and output errors in the current iteration which are considered in the fault estimating law. The proposed method using the iterative learning scheme considers state error information and fault estimating information from the previous iteration to improve the fault estimation performance in the current iteration.

(2) This method represents the first of few attempts to deal with iterative learning observer-based fault estimation problems for nonlinear uncertain systems. Unlike the conventional iterative learning schemebased fault estimation methods, this technical note designed a novel optimal function using expectation to deal with the randomly occurring parameter uncertainties.

(3) The proposed method inherits the advantages of a conventional iterative learning scheme, and LMI is used to improve the performance of fault estimation due to the accurate system model. As a result, it can reduce the computing complexity and enormously increase the efficiency and veracity of this method.

(4) The rest of this paper is organized as follows. In Section 2, the problem formulation and nonlinear system with randomly occurring parameter uncertainties are introduced. In Section 3, fault estimation using an iterative learning scheme is proposed to achieve desired fault estimation results. Then, convergence analysis based on LMI is used to solve the problem in Section 4. Simulation results are presented to illustrate the effectiveness of the proposed method in Section 5, followed by some concluding remarks in Section 6.

\section{Problem Statement and Preliminaries}

Consider the following nonlinear uncertain system

$$
\begin{aligned}
& \dot{x}(t)=\bar{A} x(t)+B u(t)+B_{g} g(x(t), t)+B_{f} f(t), \\
& y(t)=\bar{C} x(t),
\end{aligned}
$$

where $t \in[0, T]$ is the continuous-time index, $x(t) \in \mathbb{R}^{n}$ is the state vector, $y(t) \in \mathbb{R}^{p}$ is the output vector, $u(t) \in \mathbb{R}^{m}$ represents the input vector, and $f(t) \in \mathbb{R}^{q}$ stands for the fault signal. $\bar{A}=A+\alpha(t) \Delta A(t)$ and $\bar{C}=C+\beta(t) \Delta C(t)$ denote the state parameter matrix and output parameter matrix, respectively. The function $g(x(t), t) \in \mathbb{R}^{r}$ is a known nonlinear function. $A \in \mathbb{R}^{n \times n}, B \in \mathbb{R}^{n \times r}, B_{g} \in \mathbb{R}^{n \times r}, B_{f} \in \mathbb{R}^{n \times q}$, and $C \in$ $\mathbb{R}^{p \times n}$ are all constant matrices with appropriate dimensions and $n>p \geq q$. 
In this technical note, the random variables $\alpha(t)$ and $\beta(t)$ are defined to describe the parameter variations of a random nature. For system (1), the following definitions and assumptions are made available.

Definition 1. The form $\Xi\{\bullet\}$ denotes the expectation of the random variable. Meanwhile, the occurrence probability of the event is defined as $\operatorname{Prob}\{\bullet\}$.

Assumption 1. The pairs $(A, B)$ and $(A, C)$ are stabilizable and detectable, respectively.

Assumption 2. The matrices $\Delta A(t)$ and $\Delta C(t)$ represent the norm bounded parameter uncertainties of the following structure.

$$
\begin{aligned}
& \Delta A(t)=M_{1} F_{1}(t) N_{1}, \\
& \Delta C(t)=M_{2} F_{2}(t) N_{2},
\end{aligned}
$$

where $M_{i}$ and $N_{i}$ are known matrices with adequate dimensions; the unknown matrices $F_{i}(t)$ satisfy the conditions $F_{i}(t) F_{i}^{T}(t) \leq I, i=1,2$. The stochastic variables $\alpha(t)$ and $\beta(t)$ are Bernoulli distributed white sequences taking on values of either zero or one with

$$
\begin{aligned}
& \operatorname{Prob}\{\alpha(t)=1\}=\theta_{1}, \\
& \operatorname{Prob}\{\alpha(t)=0\}=1-\theta_{1}, \\
& \operatorname{Prob}\{\beta(t)=1\}=\theta_{2}, \\
& \operatorname{Prob}\{\beta(t)=0\}=1-\theta_{2},
\end{aligned}
$$

in which $\theta_{1} \in[0,1]$ and $\theta_{2} \in[0,1]$ are known constants. It is assumed that $\alpha(t)$ and $\beta(t)$ are independent of each other.

Assumption 3. The desired initial state value at each iteration is $x_{k}(0)=x(0)$ with the definition that $k$ is the iteration index.

Assumption 4. For the nonlinear term $g(x(t), t)$, there exists a known positive constant parameter $\delta_{1}$ which leads to satisfy the Lipschitz conditions. For example,

$\left\|g\left(x_{1}(t), t\right)-g\left(x_{2}(t), t\right)\right\| \leq \delta_{1}\left\|x_{1}(t)-x_{2}(t)\right\|, \quad \forall x_{1}(t), x_{2}(t) \in \mathbb{R}^{n}$,

where $\delta_{1}$ is called Lipschitz constant and $g(0, t)=0$ if the set $S=\mathbb{R}^{n}$ is globally Lipschitz.

In order to achieve the derivation of the iterative learning observer, two lemmas are introduced in this paper at first.

Lemma 1. Consider that $G$ and $H$ are constant matrices with appropriate dimensions, there exists matrix of adequacy dimensions $E(t)$ that satisfied the condition $E^{T}(t) E(t) \leq I$; for any positive scalar $\varepsilon$, the following inequality is verified [38].

$$
G E(t) H+H^{T} E^{T}(t) G^{T} \leq \varepsilon^{-1} F F^{T}+\varepsilon H^{T} H .
$$

Lemma 2 (Schur complement theorem) [39, 40]. Consider that there are two symmetric matrices $R$ and $Q$, the inequality

$$
\left[\begin{array}{ll}
Q & S \\
S^{T} & R
\end{array}\right]>0
$$

is equal to equation (7).

$$
\left\{\begin{array}{l}
R \geq 0, \\
Q-S R^{+} S^{T} \geq 0, \\
S\left(I-R R^{+}\right) \geq 0 .
\end{array}\right.
$$

In reality, noise, time delay, model uncertainties, unknown input, and sensor faults may come into the system inadvertently due to the complex environment and cumbersome process. They will affect the operation performance in different ways. For example, measurable precision of the sensor drops greatly when there is noise. Model uncertainties will influence the control precision and tracking accuracy. However, they rarely appear together. Otherwise, it makes the system breakdown and even disaster. As a result, for expression to be concise, this paper is addressed to analyze the impact of randomly occurring parameter uncertainties.

\section{Iterative Learning Observer Design}

In this section, the state observer is designed to estimate the system states and outputs, and an iterative learning law is designed for fault estimation.

Based on system (1), the observer-based fault estimator considered in this paper is proposed as

$$
\begin{aligned}
& \dot{\hat{x}}_{k}(t)=A \widehat{x}_{k}(t)+B u(t)+B_{g} g\left(\widehat{x}_{k}(t), t\right)+B_{f} \widehat{f}_{k}(t)+L\left[y(t)-\widehat{y}_{k}(t)\right] \\
& \widehat{y}_{k}(t)=C \widehat{x}_{k}(t)
\end{aligned}
$$

The order of the observer equals the number of states. In (7), $\hat{x}_{k}(t)$ and $\hat{y}_{k}(t)$ are the state estimate and output estimate of state vector $x(t)$ and output vector $y(t)$ at $k$ iterations, respectively. The parameter matrix $L$ represents the observer gain. $\widehat{f}_{k}(t)$ denotes the estimate of fault signal $f(t)$ at $k$ iterations.

On the other hand, defining that $\Delta g_{k}(t)=g\left(x_{k}(t), t\right)-$ $g\left(\hat{x}_{k}(t), t\right)$, the state estimating error $e_{k}(t)$ is shown as the following form.

$$
\begin{aligned}
\dot{e}_{k}(t)= & \dot{x}(t)-\dot{\hat{x}}_{k}(t)=(A-L C) e_{k}(t)+B_{f} r_{k}(t) \\
& +B_{g} \Delta g_{k}(t)+[\alpha(t) \Delta A-L \beta(t) \Delta C] x(t) .
\end{aligned}
$$

Then, the iterative learning scheme based on the fault estimating law is proposed as

$$
\widehat{f}_{k+1}(t)=\widehat{f}_{k}(t)+K_{1} e_{k}(t)+K_{2} \dot{e}_{k}(t),
$$


in which $K_{1}$ and $K_{2}$ stand for iterative learning gain matrices. In order to simplify the following derivation, one can give a definition of the iterative learning error of fault estimation.

$$
\begin{aligned}
r_{k+1}(t) & =f(t)-\widehat{f}_{k+1}(t)=r_{k}(t)-K_{1} e_{k}(t)-K_{2} \dot{e}_{k}(t) \\
& =Q_{1} e_{k}(t)+Q_{2} r_{k}(t)+Q_{3} x(t)+Q_{4} \Delta g_{k}(t) .
\end{aligned}
$$

In (11), the matrices are defined as $Q_{1}=-\left[K_{1}+K_{2}\right.$ $(A-L C)], Q_{2}=\left(I-K_{2} B_{f}\right), Q_{3}=-K_{2} B_{g}$, and $Q_{4}=-K_{2}[\alpha$ $(t) \Delta A-L \beta(t) \Delta C]$.

Assume that $\Theta=A-L C, \Delta \Theta=\alpha(t) \Delta A-L \beta(t) \Delta C$, and $\Delta y(t)=y(t)-\widehat{y}_{k}(t)$, then the dynamic error system will be obtained as

$$
\begin{aligned}
\dot{e}_{k}(t) & =\Theta e_{k}(t)+B_{f} r_{k}(t)+\Delta \Theta x(t), \\
\Delta y(t) & =C e_{k}(t) .
\end{aligned}
$$

\section{Convergence Analysis}

The following theorem gives the convergence of the proposed iterative learning-based observer for the case that the initial state is accurately reset. The Lyapunov function candidate is constructed to guarantee the stability of error system (12), and a novel optimal function is proposed to ensure the perfect fault tracking trajectory.

Theorem 1. Consider that a nonlinear system with randomly occurring uncertainties (1) and the iterative learning fault estimation law (10) are applied as well as Assumptions 1, 2, 3, and 4 hold. According to Lemmas 1 and 2, for scalar $\gamma$ $\in[0,1]$, the error dynamic system (12) is asymptotically stable while satisfying the fault estimating error convergence, if there exists positive-definite matrices $P=P^{T}, Q=Q^{T}$, scalar $\varepsilon_{i}>0$, $\varepsilon_{i} \varepsilon_{i}^{-1}=I$, and $i=1,2,3$, and the symmetric negative definite matrix $\Pi$ satisfies

$$
\Pi=\left[\begin{array}{ccccccccc}
-I & \Pi_{12} & \Pi_{13} & -K_{2} B_{g} & 0 & -\rho_{1} K_{2} M_{1} & 0 & K_{2} & 0 \\
* & \Pi_{22} & P B_{f} & P B_{g} & 0 & \rho_{1} P M_{1} & -\rho_{2} \widehat{L} M_{2} & 0 & C^{T} \widehat{L}^{T} \\
* & * & -\gamma^{2} I & 0 & 0 & 0 & 0 & 0 & 0 \\
* & * & * & -\lambda_{1} I & 0 & 0 & 0 & 0 & 0 \\
* & * & * & * & \Pi_{44} & \rho_{1} Q M_{1} & 0 & 0 & 0 \\
* & * & * & * & * & -\varepsilon_{1} I & 0 & 0 & 0 \\
* & * & * & * & * & * & -\varepsilon_{2} I & 0 & \rho_{2} M_{2}^{T} \widehat{L}^{T} \\
* & * & * & * & * & * & * & \varepsilon_{3}^{-1} P & 0 \\
* & * & * & * & * & * & * & * & \varepsilon_{3} P
\end{array}\right]<0,
$$

in which $\Pi_{12}=-K_{1}-K_{2} A, \Pi_{13}=I-K_{2} B_{f}, \Pi_{22}=A^{T} P-$ $C^{T} \widehat{L}^{T}+P A-\widehat{L} C, \Pi_{44}=A^{T} Q+Q A+G_{1}+G_{2}+\varepsilon_{1} N_{1}^{T} N_{1}+\varepsilon_{2}$ $N_{2}^{T} N_{2}$, and $\widehat{L}=P L$. Then, the observer gain matrix can be obtained as $L=P^{-1} \widehat{L}$. Theorem 1 presents the sufficient condition for the existence and design of the iterative learning fault estimator for system (1). It should point out that (13) does not have a feasible solution for the existence of nonlinear terms. As a result, the following remarks are introduced to improve the application of the proposed method.

Remark 1. To linearize nonlinear term $-\gamma^{2} I$ in (13), the following equation is defined:

$$
\varphi=-\gamma^{2} I
$$

Remark 2. In (13), there exist nonlinear terms $\varepsilon_{3}^{-1} P$ and $\varepsilon_{3} P$; we use the following constraint and approximation

$$
\begin{gathered}
P>\varsigma_{1} I, \\
\varepsilon_{3}+\frac{1}{\varepsilon_{3}} \geq 2 .
\end{gathered}
$$

Then, a new LMI is constructed of $\alpha$ and $\beta=\alpha \varepsilon_{3}$. From (15), we have $1 / \varepsilon_{3} P \geq 2 P-\varepsilon_{3} P=\left(2 \varsigma_{1}-\varsigma_{2}\right) I$ and $\varepsilon_{3} P \geq \varepsilon_{3} \varsigma_{1} I$ $=\varsigma_{2} I$. Hence, the terms $\left(2 \varsigma_{1}-\varsigma_{2}\right) I$ and $\varsigma_{2} I$ are used to replace the blocks $1 / \varepsilon_{3} P$ and $\varepsilon_{3} P$ in (13), respectively.

Corollary 1. According to Remarks 1 and 2, Theorem 1 can be rewritten as the following optimal functions subject to the LMI in (16) if there exist symmetric positive definite matrixes $P=P^{T}>0, Q=Q^{T}>0$, scalars $\varsigma_{1}>0$ and $\varsigma_{2}>0, \varphi \in[0,1]$, and $\varepsilon_{i}>0, i=1,2$. 
Min $\{\varphi\}$,

s.t. $\quad \Pi=\left[\begin{array}{ccccccccc}-I & \Pi_{12} & \Pi_{13} & -K_{2} B_{g} & 0 & -\rho_{1} K_{2} M_{1} & 0 & K_{2} & 0 \\ * & \Pi_{22} & P B_{f} & P B_{g} & 0 & \rho_{1} P M_{1} & -\rho_{2} \widehat{L} M_{2} & 0 & C^{T} \widehat{L}^{T} \\ * & * & -\varphi I & 0 & 0 & 0 & 0 & 0 & 0 \\ * & * & * & -\lambda_{1} I & 0 & 0 & 0 & 0 & 0 \\ * & * & * & * & \Pi_{44} & \rho_{1} Q M_{1} & 0 & 0 & 0 \\ * & * & * & * & * & -\varepsilon_{1} I & 0 & 0 & 0 \\ * & * & * & * & * & * & -\varepsilon_{2} I & 0 & \rho_{2} M_{2}^{T} \widehat{L}^{T} \\ * & * & * & * & * & * & * & \Pi_{77} & 0 \\ * & * & * & * & * & * & * & * & -\varsigma_{2} P\end{array}\right]<0$,

where $\Pi_{77}=-\left(2 \varsigma_{1}-\varsigma_{2}\right) I$. Feasible solutions will be obtained through the LMI toolbox in Matlab. Then, the fault estimation algorithm (10) using the iterative learning scheme can realize $e_{k}(t)$ and $r_{k}(t)$ uniformly bounded. Namely, the monotonic convergence of tracking error (11) and the Ho performance of the error-argued system (12) are achieved. As in the discussion above, Corollary 1 can be employed for fault estimator design of nonlinear uncertain system (1) directly.

Remark 3. In Corollary 1, one can find out that the theoretical results are related only with the random distribution while being free of the types of uncertainties. Hence, the proposed scheme achieves a high generality and it could be integrated into both constant uncertain case and time-varying uncertain case.

Proof 1. The first objective of this technical note is to achieve the stability and convergence of the state observer, to realize the desired state estimating results. Consider the Lyapunov function as

$$
V(t)=e_{k}^{T}(t) P e_{k}(t)+x^{T}(t) Q x(t)>0 .
$$

From (10) and (12), one can calculating the derivative of $V(t)$ with respect to time as

$$
\begin{aligned}
\dot{V}(t)= & \dot{e}_{k}^{T}(t) P e_{k}(t)+e_{k}^{T}(t) P \dot{e}_{k}(t)+\dot{x}^{T}(t) Q x(t)+x^{T}(t) Q \dot{x}(t) \\
= & e_{k}^{T}(t)(A-L C)^{T} P e_{k}(t)+x^{T}(t)[\alpha(t) \Delta A-L \beta(t) \Delta C]^{T} P e_{k}(t) \\
& +e_{k}^{T}(t) P(A-L C) e_{k}(t)+e_{k}^{T}(t) P[\alpha(t) \Delta A-L \beta(t) \Delta C] x(t) \\
& +r_{k}^{T}(t) B_{f}^{T} P e_{k}(t)+e_{k}^{T}(t) P B_{f} r_{k}(t)+\Delta g_{k}^{T}(t) B_{g}^{T} P e_{k}(t) \\
& +e_{k}^{T}(t) P B_{g} \Delta g_{k}(t)+x^{T}(t)(A+\alpha(t) \Delta A)^{T} Q x(t) \\
& +u^{T}(t) B^{T} Q x(t)+f^{T}(t) B_{f}^{T} Q x(t) \\
& +x^{T}(t) Q(A+\alpha(t) \Delta A) x(t)+x^{T}(t) Q B u(t) \\
& +x^{T}(t) Q B_{f} f(t) .
\end{aligned}
$$

For two real symmetric positive definite symmetric matrixes $G_{1}$ and $G_{2}$, the following inequalities are established as

$$
\begin{aligned}
u^{T}(t) B^{T} Q x(t) & +x^{T}(t) Q B u(t) \leq x^{T}(t) G_{1} x(t) \\
& +u^{T}(t) B^{T} Q G_{1}^{-1} Q B u(t) \leq x^{T}(t) G_{1} x(t) \\
& +u_{1} \lambda_{1 \_ \text {max }}\left(B^{T} Q G^{-1} Q B\right), \\
f^{T}(t) B_{f}^{T} Q x(t) & +x^{T}(t) Q B_{f} f(t) \leq x^{T}(t) G_{2} x(t) \\
& +f^{T}(t) B_{f}^{T} Q G_{2}^{-1} Q B_{f} f(t) \leq x^{T}(t) G_{2} x(t) \\
& +f_{1} \lambda_{2 \_ \text {max }}\left(B_{f}^{T} Q G_{2}^{-1} Q B_{f}\right) .
\end{aligned}
$$

One can further obtain that

$$
\dot{V}(t) \leq \xi_{k}^{T}(t) \Pi_{1} \xi(t)+\delta_{1}+\delta_{2},
$$

where

$$
\begin{gathered}
\xi_{k}(t)=\left[\begin{array}{c}
e_{k}(t) \\
r_{k}(t) \\
\Delta g_{k}(t) \\
x(t)
\end{array}\right], \\
\Pi_{1}=\left[\begin{array}{cccc}
\Theta^{T} P+P \Theta & P B_{f} & P B_{g} & P \Delta \Theta \\
* & 0 & 0 & 0 \\
* & * & 0 & 0 \\
* & * & * & \Pi_{1 \_44}
\end{array}\right] . \\
\text { and } \delta_{2}=f_{1} \lambda_{2 \_ \text {max }}\left(B_{f}^{T} Q G_{2}^{-1} Q B_{f}\right) \text {. Based on the Lyapunov }
\end{gathered}
$$


stability theory, the error dynamic system is stable and the designed observer is converged if the inequalities $V(t)>0$ and $\dot{V}(t)<0$ hold. Based on Lemma 2 , it is obvious that the inequality $\dot{V}(t)<0$ holds only if the equation $\left\|\xi_{k}^{T}(t)\right\|^{2}>\delta_{1}+\delta_{2} / \phi$ is true and $\Pi_{1}<0$.

Notice that the Lyapunov function is constructed to only ensure the stability of the output when the updating law is applied in system (1). The second objective in this paper is to obtain appropriate learning gain matrixes such that the tracking error converges to zero for all within the whole time interval $t$ as $[0, T]$. Moreover, in the sense of randomly occurring uncertainties, a novel optimal function of the expectation form is proposed to ensure the convergence of fault estimation. To attain $H \infty$ robustness performance and convergence of the proposed method, the following performance index is introduced for the prescribed scalar $\gamma \in[0,1]$ at any iteration $k \in \mathrm{Z}_{+}$.

$$
J_{1}=\Xi\left[\int_{0}^{\tau}\left[r_{k+1}^{T}(t) r_{k+1}(t)-\gamma^{2} r_{k}^{T}(t) r_{k}(t)\right] d t\right] \leq 0 .
$$

Using Assumption 4, there exists a positive scalar $\lambda_{1}$ $\epsilon[0,1]$ that satisfies

$$
J_{2}=\int_{0}^{t}\left[\lambda_{1} \delta_{1} e_{k}^{T}(t) e_{k}(t)-\lambda_{1} \Delta g_{k}^{T}(t) \Delta g_{k}(t)\right] d t \geq 0 .
$$

Then the derivative of $V(t)$ and the inequality (23) are taken into (22); the optimal function $J_{1}$ is rewritten as follows:

$$
J_{1}<J_{1}+J_{2}
$$

Denoting that $J=J_{1}+J_{2}$, then one can get that

$$
\begin{aligned}
J & =\Xi\left[J_{1}+J_{2}\right]=\Xi\left[J_{1}+J_{2}+\int \dot{V}(t) d t-[V(\tau)-V(0)]\right] \\
& =\Xi\left[\int_{0}^{\tau} \xi_{k}^{T}(t) \Pi_{2} \xi_{k}(t) d t-[V(\tau)-V(0)]\right] \leq 0 .
\end{aligned}
$$

For convenience of later analysis, the expectations of random terms are defined as $\Xi\{\alpha(t)\}=\rho_{1}, \Xi\{\beta(t)\}=\rho_{2}$. Based on Lemma 2 and (25), one can obtain that

$\Pi_{2}=\left[\begin{array}{cccc}\Pi_{2 \_11}+Q_{1}^{T} Q_{1} & P B_{f}+Q_{1}^{T} Q_{2} & P B_{g}+Q_{1}^{T} Q_{3} & \Pi_{2 \_14} \\ * & -\gamma^{2} I+Q_{2}^{T} Q_{2} & Q_{2}^{T} Q_{3} & Q_{2}^{T} Q_{4} \\ * & * & -\lambda_{1} I+Q_{3}^{T} Q_{3} & Q_{3}^{T} Q_{4} \\ * & * & * & \Pi_{1-33}+Q_{4}^{T} Q_{4}\end{array}\right]<0$,

where $\Pi_{2 \_11}=\Theta^{T} P+P \Theta+Q_{1}^{T} Q_{1}$ and $\Pi_{2 \_14}=P\left[\rho_{1} \Delta A-L \rho_{2}\right.$ $\Delta C]+Q_{1}^{T} Q_{3}$. According to Lemma 2 , it is easy to see that (26) holds if the following inequality holds:

$$
\Pi_{3}=\left[\begin{array}{ccccc}
-I & Q_{1} & Q_{2} & Q_{3} & Q_{4} \\
* & \Theta^{T} P+P \Theta & P B_{f} & P B_{g} & P\left[\rho_{1} \Delta A-L \rho_{2} \Delta C\right] \\
* & * & -\gamma^{2} I & 0 & 0 \\
* & * & * & -\lambda_{1} I & 0 \\
* & * & * & * & \Pi_{1 \_44}
\end{array}\right]<0 .
$$

Then, matrix $\Pi_{3}$ is well extracted into the summation of two components. One is the constant term, and another is the uncertain term.

$$
\begin{aligned}
& \Pi_{3}=\underbrace{}_{\left.\begin{array}{ccccc}
-I & Q_{1} & Q_{2} & Q_{3} & 0 \\
* & \Theta^{T} P+P \Theta & P B_{f} & P B_{g} & 0 \\
* & * & -\gamma^{2} I & 0 & 0 \\
* & * & * & -\lambda_{1} I & 0 \\
* & * & * & * & A^{T} Q+Q A+G_{1}+G_{2}
\end{array}\right]} \\
&+\left[\begin{array}{ccccc}
0 & 0 & 0 & 0 & -K_{2}\left(\rho_{1} \Delta A-L \rho_{2} \Delta C\right) \\
* & 0 & 0 & 0 & P\left[\rho_{1} \Delta A-L \rho_{2} \Delta C\right] \\
* & * & 0 & 0 & 0 \\
* & * & * & 0 & 0 \\
* & * & * & * & \rho_{1} \Delta A^{T} Q+\rho_{1} Q \Delta A
\end{array}\right]
\end{aligned}
$$

By expanding the uncertain term $\Pi_{5}$ with (2), one has

$$
\Pi_{5}=-\left[\begin{array}{c}
-\rho_{1} K_{2} M_{1} \\
\rho_{1} P M_{1} \\
0 \\
0 \\
\rho_{1} Q M_{1}
\end{array}\right] F_{1}\left[0000 N_{1}\right]+\left[\begin{array}{c}
\rho_{2} K_{2} L M_{2} \\
-\rho_{2} P L M_{2} \\
0 \\
0 \\
0
\end{array}\right] F_{2}\left[0000 N_{2}\right]
$$

$$
\begin{aligned}
& -\left[\begin{array}{c}
0 \\
0 \\
0 \\
0 \\
N_{1}^{T}
\end{array}\right] F_{1}^{T}\left[-\rho_{1} M_{1}^{T} K_{2}^{T} \rho_{1} M_{1}^{T} P 00 \rho_{1} M_{1}^{T} Q\right] \\
& +\left[\begin{array}{c}
0 \\
0 \\
0 \\
0 \\
N_{2}^{T}
\end{array}\right] F_{2}^{T}\left[\rho_{2} M_{2}^{T} L K_{2}^{T}-\rho_{2} M_{2}^{T} L^{T} P 000\right] .
\end{aligned}
$$


Moreover, using Lemma 1, the inequality (26) holds if and only if there exists $\varepsilon_{1}>0$ and $\varepsilon_{2}>0$ such that

$$
\begin{aligned}
& \Pi_{5} \leq \varepsilon_{1}^{-1}\left[\begin{array}{c}
-\rho_{1} K_{2} M_{1} \\
\rho_{1} P M_{1} \\
0 \\
0 \\
\rho_{1} Q M_{1}
\end{array}\right]\left[\begin{array}{c}
-\rho_{1} K_{2} M_{1} \\
\rho_{1} P M_{1} \\
0 \\
0 \\
\rho_{1} Q M_{1}
\end{array}\right]^{T}+\varepsilon_{1}\left[\begin{array}{c}
0 \\
0 \\
0 \\
0 \\
N_{1}^{T}
\end{array}\right]\left[\begin{array}{c}
0 \\
0 \\
0 \\
0 \\
N_{1}^{T}
\end{array}\right]^{T} \\
& +\varepsilon_{2}^{-1}\left[\begin{array}{c}
\rho_{2} K_{2} L M_{2} \\
-\rho_{2} P L M_{2} \\
0 \\
0 \\
0
\end{array}\right]\left[\begin{array}{c}
\rho_{2} K_{2} L M_{2} \\
-\rho_{2} P L M_{2} \\
0 \\
0 \\
0
\end{array}\right]^{T} \\
& +\varepsilon_{2}\left[\begin{array}{c}
0 \\
0 \\
0 \\
0 \\
N_{2}^{T}
\end{array}\right]\left[\begin{array}{c}
0 \\
0 \\
0 \\
N_{2}^{T}
\end{array}\right]^{T}
\end{aligned}
$$

With Lemma 2, (27) can be further written as

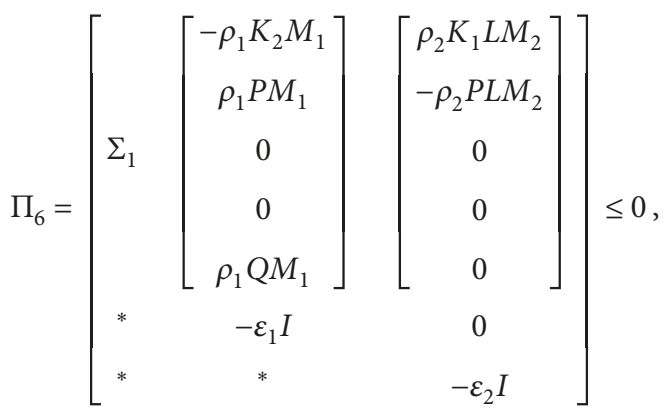

where

$$
\begin{gathered}
\Sigma_{1}=\left[\begin{array}{ccccc}
-I & Q_{1} & Q_{2} & Q_{3} & 0 \\
* & \Theta^{T} P+P \Theta & P B_{f} & P B_{g} & 0 \\
* & * & -\gamma^{2} I & 0 & 0 \\
* & * & * & -\lambda_{1} I & 0 \\
* & * & * & * & \Sigma_{1 \_55}
\end{array}\right], \\
\Sigma_{1 \_55}=A^{T} Q+Q A+G_{1}+G_{2}+\varepsilon_{1} N_{1}^{T} N_{1}+\varepsilon_{2} N_{2}^{T} N_{2} .
\end{gathered}
$$

Similarly, expanding $\Pi_{6}$, one has

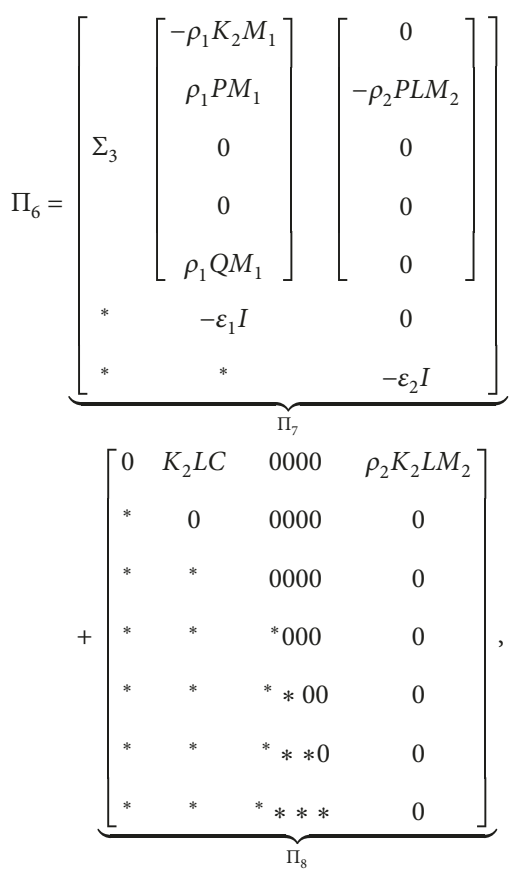

in which

$$
\Sigma_{3}=\left[\begin{array}{ccccc}
-I & -\left(K_{1}+K_{2} A\right) & Q_{2} & Q_{3} & 0 \\
* & \Theta^{T} P+P \Theta & P B_{f} & P B_{g} & 0 \\
* & * & -\gamma^{2} I & 0 & 0 \\
* & * & * & -\lambda_{1} I & 0 \\
* & * & * & * & \Sigma_{1 \_55}
\end{array}\right] .
$$

By denoting $\widehat{L}=P L$, then one can get that $L=P^{-1} \widehat{L}$. Using Lemma 1 and letting $\bar{P}=P^{-1}$, inequalities can immediately be achieved for $\varepsilon_{3}>0$.

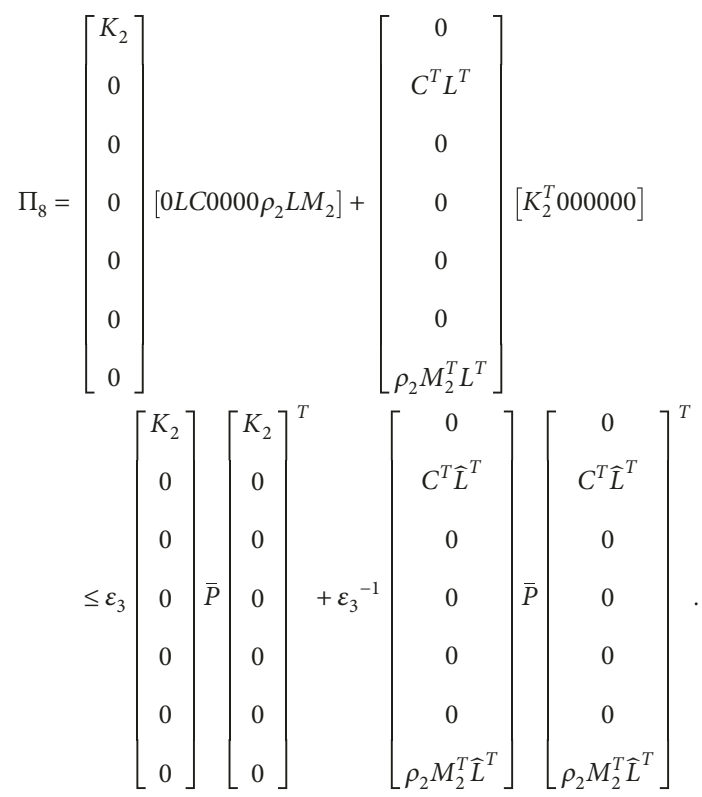


Hence, Theorem 1 is obtained by employing Lemma 2 . This completes the proof.

\section{Illustrative Example}

In this section, a numerical example has been performed to demonstrate the validity and effectiveness of the proposed approach.

Consider the following nonlinear systems (36) with randomly occurring parameter uncertainties according to (1), where the state variables at $k$ iteration is denoted by $x_{k}(t)=$ $\left[x_{1, k}(t), x_{2, k}(t), x_{3, k}(t), x_{4, k}(t)\right]^{T}$ :

$$
\begin{aligned}
\dot{x}_{k}(t)= & (A+\alpha(t) \Delta A(t)) x_{k}(t)+B u_{k}(t) \\
& +B_{g} g\left(x_{k}(t), t\right)+B_{f} f(t), \\
y_{k}(t)= & (C+\beta(t) \Delta C(t)) x_{k}(t) .
\end{aligned}
$$

The constant matrixes are given by

$$
\begin{aligned}
A & =\left[\begin{array}{cccc}
0 & 1 & 0 & 0 \\
0 & 0 & 1 & 0 \\
-21.6 & -13.6 & -4.2 & 16 \\
-1 & 0 & 0 & 0
\end{array}\right], \\
B & =\left[\begin{array}{c}
0 \\
0.2471 \\
0 \\
-0.4758
\end{array}\right], \\
B_{f} & =\left[\begin{array}{c}
0 \\
0.5 \\
0 \\
-0.5
\end{array}\right] \\
C & =\left[\begin{array}{c}
0010 \\
0100
\end{array}\right]
\end{aligned}
$$

and the nonlinear term is described as

$$
B_{g}=\left[\begin{array}{c}
0.1 \\
0 \\
0 \\
0
\end{array}\right] \text {, }
$$

$$
g\left[x_{k}(t), t\right]=\sin \left(x_{1, k}(t)\right) \text {. }
$$

The initial desired value of state variables is set to be $x_{d}$ $(0)=\left[\begin{array}{llll}0 & 0 & 0 & 0\end{array}\right]^{T}$ and the controller that is employed as constant $u_{k}(t)=\left[\begin{array}{llll}1 & 1 & 1 & 1\end{array}\right]^{T}$; the sampling period is $T$ $=0.1$. The following three cases of fault signals $f(t)=f_{1}(t)$, $f(t)=f_{2}(t)$, and $f(t)=f_{3}(t)$ affecting the system output behaviors are considered in this technical note.

Case 1 (sinusoidal fault signal).

$$
f_{1}(t)=\left\{\begin{array}{cc}
0.5 \sin (2 \pi t), & t \in[0,1 \mathrm{~s}), \\
\sin (2 \pi t), & t \in[1 \mathrm{~s}, 2 \mathrm{~s}), \\
1.5 \sin (2 \pi t), & t \in[2 \mathrm{~s}, 3 \mathrm{~s}), \\
2 \sin (2 \pi t), & t \in[3 \mathrm{~s}, 4 \mathrm{~s}), \\
2.5 \sin (2 \pi t), & t \in[4 \mathrm{~s}, 5 \mathrm{~s}) .
\end{array}\right.
$$

Case 2 (constant fault signal).

$$
f_{2}(t)=\left\{\begin{array}{cc}
0.5, & t \in[0,1 \mathrm{~s}) \\
1, & t \in[1 \mathrm{~s}, 2 \mathrm{~s}) \\
1.5, & t \in[2 \mathrm{~s}, 3 \mathrm{~s}) \\
2, & t \in[3 \mathrm{~s}, 4 \mathrm{~s}) \\
2.5, & t \in[4 \mathrm{~s}, 5 \mathrm{~s})
\end{array}\right.
$$

Case 3 (intermittent fault signal).

$$
f_{3}(t)=\left\{\begin{array}{c}
|\sin (\pi t)|, \quad t \in[0,1 \mathrm{~s}), \\
|2 \sin (2 \pi t)|, \quad t \in[1 \mathrm{~s}, 2 \mathrm{~s}), \\
0, \quad t \in[2 \mathrm{~s}, 3 \mathrm{~s}), \\
|\sin (\pi t)|, \quad t \in[3 \mathrm{~s}, 4 \mathrm{~s}), \\
|2 \sin (2 \pi t)|, \quad t \in[4 \mathrm{~s}, 5 \mathrm{~s})
\end{array}\right.
$$

In the simulation, the randomly occurring uncertainties (2) with probability distribution (3) are addressed for demonstrating the effectiveness of the iterative learning fault estimator. The constant matrices of uncertainties for $\Delta A(t)$ and $\Delta C(t)$ are given as

$$
\begin{aligned}
& M_{1}=\left[\begin{array}{c}
0 \\
0.1 \\
0 \\
0.1
\end{array}\right], \\
& M_{2}=\left[\begin{array}{l}
10 \\
01
\end{array}\right], \\
& N_{1}=[0.020 .010 \cdot 010.01], \\
& N_{2}=\left[\begin{array}{l}
0.20 \cdot 10.10 .2 \\
0.10 \cdot 20.20 .1
\end{array}\right] .
\end{aligned}
$$

Moreover, the probability distribution is described as $\mathrm{Pr}$ $\mathrm{ob}\{\alpha(t)=1\}=0.6, \operatorname{Prob}\{\alpha(t)=0\}=0.4, t \in\left[0, T_{d}\right]$ and Pro 


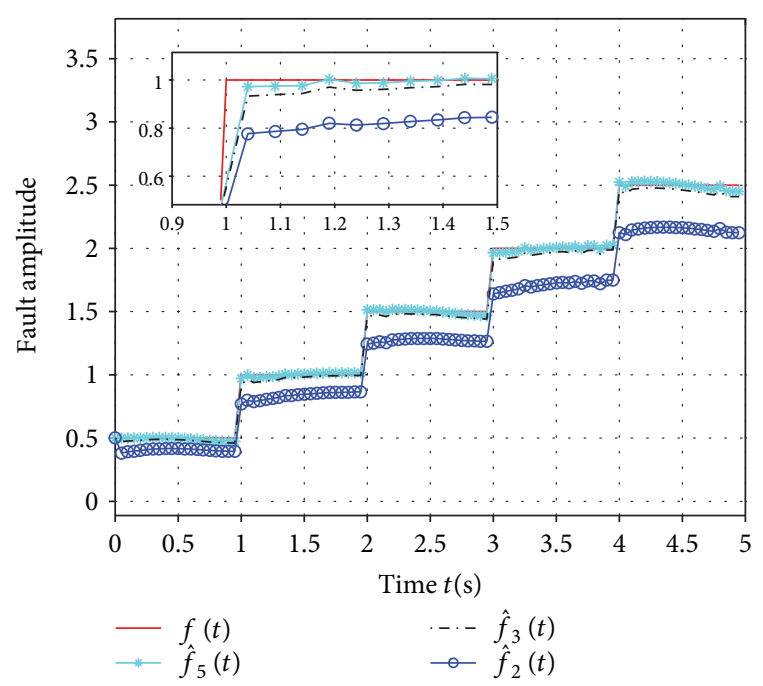

Figure 1: The tracking trajectory of abrupt fault for a nonlinear system with constant parameter uncertainties.

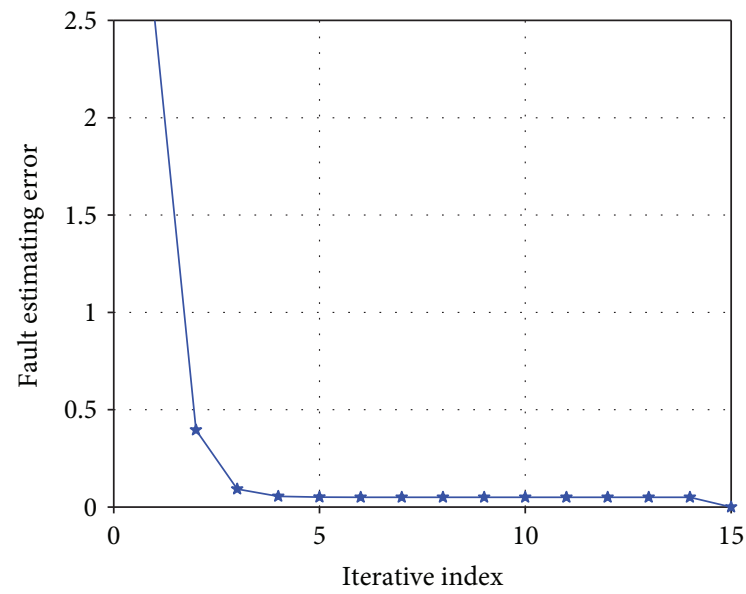

FIGURE 2: The tracking trajectory of slow variation fault for a nonlinear system with constant parameter uncertainties.

$\mathrm{b}\{\beta(t)=1\}=0.9, \operatorname{Prob}\{\beta(t)=0\}=0.1, t \in\left[0, T_{d}\right]$, in which $\bullet(t)=1$ denotes the fault occurring and $\bullet(t)=0$ represents there is no inverse fault $(\bullet$ is $\alpha$ or $\beta)$.

To further illustrate the effectiveness of the proposed fault estimation approach in a class of nonlinear uncertain systems, the maximum value of absolute error $E_{k}$ is introduced to evaluate the effectiveness of fault estimating performance in different iterations. The definition of $E_{k}$ is shown as follows.

$$
E_{k}=\sup _{t \in\left[0, T_{d}\right]}\left|f(t)-\widehat{f}_{k}(t)\right|
$$

Denoting that $\varsigma_{1}=2, \varsigma_{2}=3, \varphi=0.5, \lambda_{1}=0.1, \varepsilon_{1}=0.8$, and $\varepsilon_{2}=0.3$ and letting $G_{1}=G_{2}$, by solving the optimization problem under LMI constraints in Theorem 1, results

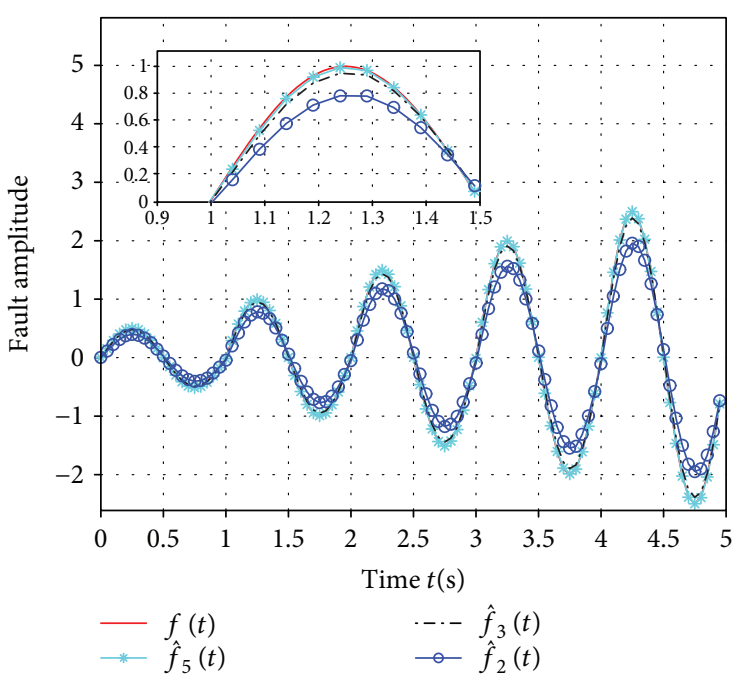

FIgURE 3: The tracking trajectory of intermittent fault for a nonlinear system with constant parameter uncertainties.

of the observer and fault estimator gain matrices are shown as follows.

$$
\begin{aligned}
& P=\left[\begin{array}{cccc}
1.8394 & 0.6114 & 0.2447 & -1.1955 \\
0.6114 & 0.6373 & 0.1154 & -0.6696 \\
0.2447 & 0.1154 & 0.0858 & -0.1665 \\
-1.1955 & -0.6696 & -0.1665 & 1.2815
\end{array}\right], \\
& Q=\left[\begin{array}{cccc}
11.4011 & 5.3333 & 0.8285 & -8.8311 \\
5.3333 & 3.4603 & 0.5080 & -4.4231 \\
0.8285 & 0.5080 & 0.2193 & -0.4700 \\
-8.8311 & -4.4231 & -0.4700 & 8.5802
\end{array}\right] \text {, } \\
& G=\left[\begin{array}{cccc}
3.9136 & 1.5742 & 0.8356 & -2.4157 \\
1.5742 & 0.9316 & 0.3007 & -0.5092 \\
0.8356 & 0.3007 & 0.2809 & -0.3881 \\
-2.4157 & -0.5092 & -0.3881 & 1.9898
\end{array}\right], \\
& K_{1}=\left[\begin{array}{llll}
-0.2873 & 0.3319 & -0.6793 & -0.4973
\end{array}\right] \text {, } \\
& K_{2}=\left[\begin{array}{llll}
0.0001 & 0.7516 & 0.0000 & -0.7489
\end{array}\right] \text {, } \\
& L=\left[\begin{array}{cc}
-0.0721 & -0.0373 \\
-0.0021 & 0.2055 \\
0.2646 & -0.0010 \\
0.0173 & 0.0711
\end{array}\right]
\end{aligned}
$$

For definiteness and without loss of generality, considering that $F_{1}(t)=0.5 \sin (2 \pi t)$ and $F_{2}(t)=\cos (\pi t)$, the fault estimation results in the nonlinear system with constant randomly occurring parameter uncertainties are shown in Figures 1-6. 


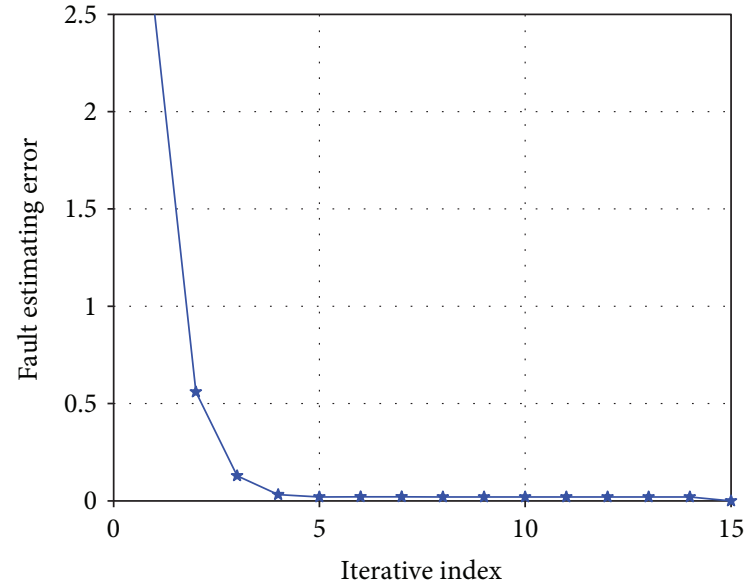

FIGURE 4: The tracking trajectory of abrupt fault for a nonlinear system with time-varying parameter uncertainties.

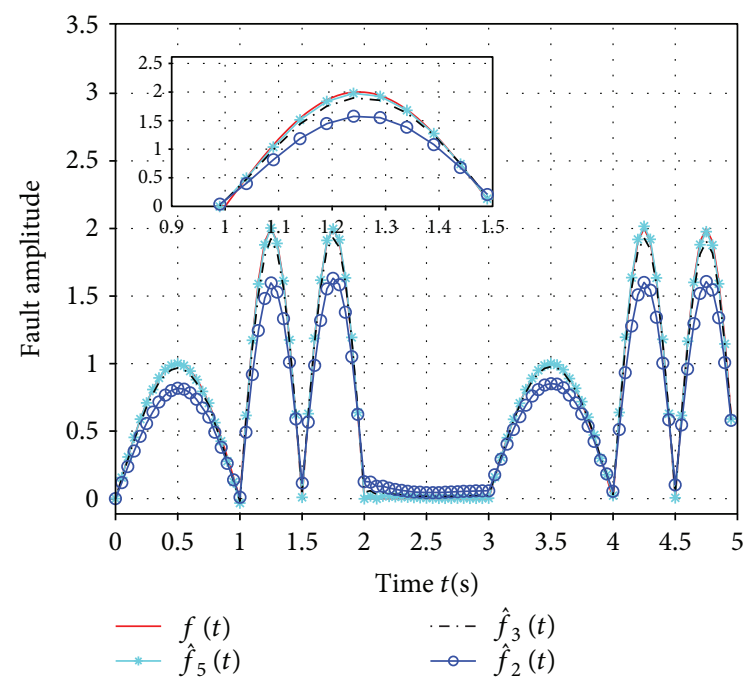

FIGURE 5: The tracking trajectory of slow variation fault for a nonlinear system with time-varying parameter uncertainties.

Figures 1, 3, and 5 show the fault estimating results and actual fault signals of constant fault, time-varying fault, and intermittent fault, respectively, in which $f(t), \widehat{f}_{2}(t), \widehat{f}_{3}(t)$, and $\widehat{f}_{5}(t)$ represent actual fault signal and estimated fault signals at the second, third, and fifth iterations, respectively. The fault estimating results are more close to the actual fault with iterations increasing. The estimated fault signal at the fifth iteration has plenty of overlaps with the actual fault signal. One can see that constant fault, time-varying fault, and intermittent fault are estimated with good accuracy. It can be concluded that the proposed fault estimation observer and algorithm have an excellent performance to estimate the actual fault.

The variation trend of maximum absolute error is exhibited in Figures 2, 4, and 6, respectively. It can be seen that decreases with iterations increase and converge to zero. One can conclude that a satisfactory estimation performance

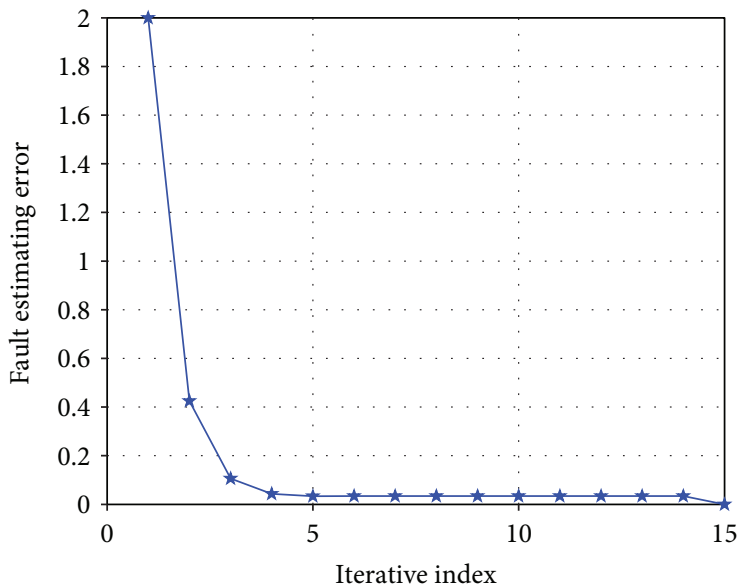

FIGURE 6: The tracking trajectory of intermittent fault for a nonlinear system with time-varying parameter uncertainties.

has been achieved. It should be pointed that the state estimating error and fault estimating results in previous iterations are utilized in the current iteration to improve the estimation performance. Compared with the conventional observerbased fault estimation approaches, the proposed method has a better performance after few iterations.

\section{Conclusion}

This paper presents a novel observer-based fault estimation method using an iterative leaning scheme for nonlinear uncertain systems where parameter uncertainties are randomly occurring. Firstly, a state observer is constructed to monitor the system status and the Lyapunov function is utilized to ensure the stability of the system. After providing the design problem of a robust monotonical convergence for the error system, an optimal function using expectation is presented to ensure the iterative learning law is applicable to systems. Meanwhile, two lemmas and two reasonable assumptions are utilized to linearize the nonlinear terms in our initial results. The LMI toolbox is utilized to obtain the results of the learning gain. Finally, the theoretical results have been verified through simulation tests. In addition, it is shown that the proposed fault estimation approach can be applicable to more general nonlinear uncertain systems.

\section{Data Availability}

The data used to support the findings of this study are included within the article.

\section{Conflicts of Interest}

The authors declare that they have no conflicts of interest.

\section{Acknowledgments}

This work was funded by the National Natural Science Foundation of China (61374135 and U1637107) and Chongqing University Postgraduates Innovation Project (CYB15051). 
All data generated or analysed during this study are included in this published article.

\section{References}

[1] D. Wu and Y. Li, "Fault diagnosis of variable pitch for wind turbines based on the multi-innovation forgetting gradient identification algorithm," Nonlinear Dynamics, vol. 79, no. 3, pp. 2069-2077, 2015.

[2] R. J. Patton and J. Chen, "Review of parity space approaches to fault diagnosis for aerospace systems," Journal of Guidance, Control, and Dynamics, vol. 17, no. 2, pp. 278-285, 1994.

[3] Z. Gao, C. Cecati, and S. X. Ding, "A survey of fault diagnosis and fault-tolerant techniques-part I: fault diagnosis with model-based and signal-based approaches," IEEE Transactions on Industrial Electronics, vol. 62, no. 6, pp. 3757-3767, 2015.

[4] C. Edwards, H. Alwi, and C. Tan, "Sliding mode methods for fault detection and fault tolerant control with application to aerospace systems," International Journal of Applied Mathematics and Computer Science, vol. 22, no. 1, pp. 109-124, 2012.

[5] Z. Wang, L. Liu, H. Zhang, and G. Xiao, "Fault-tolerant controller design for a class of nonlinear MIMO discretetime systems via online reinforcement learning algorithm," IEEE Transactions on Systems, Man, and Cybernetics: Systems, vol. 46, no. 5, pp. 611-622, 2016.

[6] Y.-X. Li and G.-H. Yang, "Robust fuzzy adaptive fault-tolerant control for a class of nonlinear systems with mismatched uncertainties and actuator faults," Nonlinear Dynamics, vol. 81, no. 1-2, pp. 395-409, 2015.

[7] K. Zhang, B. Jiang, S. Peng, and J. Xu, "Fault estimation observer design for discrete-time systems infinite-frequency domain: fault estimation observer design," International Journal of Robust and Nonlinear Control, vol. 25, no. 9, pp. 13791398, 2014.

[8] S. M. Tabatabaeipour and T. Bak, "Robust observer-based fault estimation and accommodation of discrete-time piecewise linear systems," Journal of the Franklin Institute, vol. 351, no. 1, pp. 277-295, 2014.

[9] Z. Hu, G. Zhao, L. Zhang, and D. Zhou, "Fault estimation for nonlinear dynamic system based on the second-order sliding mode observer," Circuits, Systems, and Signal Processing, vol. 35, no. 1, pp. 101-115, 2016.

[10] Z. Wang, M. Rodrigues, D. Theilliol, and Y. Shen, “Actuator fault estimation observer design for discrete-time linear parameter-varying descriptor systems," International Journal of Adaptive Control and Signal Processing, vol. 29, no. 2, pp. 242-258, 2015.

[11] B. Jiang and F. N. Chowdhury, "Fault estimation and accommodation for linear mimo discrete-time systems," IEEE Transactions on Control Systems Technology, vol. 13, no. 3, pp. 493-499, 2005.

[12] K. Zhang, B. Jiang, and V. Cocquempot, "Adaptive observerbased fast fault estimation," International Journal of Control, Automation, and Systems, vol. 6, no. 3, pp. 320-326, 2008.

[13] M. Zhong, S. X. Ding, Q.-L. Han, and Q. Ding, "Parity spacebased fault estimation for linear discrete time-varying systems," IEEE Transactions on Automatic Control, vol. 55, no. 7, pp. 1726-1731, 2010.

[14] Z. Wang, Y. Shen, and X. Zhang, "Actuator fault estimation for a class of nonlinear descriptor systems," International Journal of Systems Science, vol. 45, no. 3, pp. 487-496, 2014.
[15] M. Witczak, M. Buciakowski, V. Puig, D. Rotondo, and F. Nejjari, "An LMI approach to robust fault estimation for a class of nonlinear systems," International Journal of Robust and Nonlinear Control, vol. 26, no. 7, pp. 1530-1548, 2016.

[16] M. Shahriari-Kahkeshi, F. Sheikholeslam, and J. Askari, "Adaptive fault detection and estimation scheme for a class of uncertain nonlinear systems," Nonlinear Dynamics, vol. 79, no. 4, pp. 2623-2637, 2015.

[17] Q. Zhang, Y. Liu, and Z. Zhang, "A new optimization method for sheet metal forming processes based on an iterative learning control model," The International Journal of Advanced Manufacturing Technology, vol. 85, no. 5-8, pp. 1063-1075, 2016.

[18] W. Xiong, X. Yu, R. Patel, and W. Yu, "Iterative learning control for discrete- time systems with event-triggered transmission strategy and quantization," Automatica, vol. 72, pp. 84-91, 2016.

[19] G. Cheng, Y. L. Cheng, L. H. Shen, J. B. Qiu, and S. Zhang, "Gear fault identification based on Hilbert huang transform and SOM neural network," Measurement, vol. 46, no. 3, pp. 1137-1146, 2013.

[20] N. Kocyigit, "Fault and sensor error diagnostic strategies for a vapor compression refrigeration system by using fuzzy inference systems and artificial neural network," International Journal of Refrigeration, vol. 50, pp. 69-79, 2015.

[21] A. Yadav and Y. Dash, "An overview of transmission line protection by artificial neural network: fault detection, fault classification, fault location, and fault direction discrimination," Advances in Artificial Neural Systems, vol. 2014, Article ID 230382, 20 pages, 2014.

[22] W. Cao, W. Cong, and M. Sun, "Iterative learning based fault detection and estimation in nonlinear systems," Journal of Systems Engineering and Electronics, vol. 23, no. 3, pp. 419424, 2012.

[23] B. Yan, H. Wang, and H. Wang, "A novel approach to fault diagnosis for time-delay systems," Computers \& Electrical Engineering, vol. 40, no. 7, pp. 2273-2284, 2014.

[24] J. Shi, X. He, and D. Zhou, "Iterative learning based estimation of periodically occurring faults," IET Control Theory \& Applications, vol. 10, no. 2, pp. 244-251, 2016.

[25] B. Yan, H. Su, and W. Ma, "Fault detection and identification for a class of nonlinear systems with model uncertainty," Applied Mathematical Modelling, vol. 40, no. 15-16, pp. 7368-7381, 2016.

[26] L. Wang, B. Liu, J. Yu, P. Li, R. Zhang, and F. Gao, "Delay-range-dependent-based hybrid iterative learning fault-tolerant guaranteed cost control for multiphase batch processes," Industrial and Engineering Chemistry Research, vol. 57, no. 8, pp. 2932-2944, 2018.

[27] L. Wang, Y. Shen, B. Li, J. Yu, R. Zhang, and F. Gao, "Hybrid iterative learning fault-tolerant guaranteed cost control design for multi-phase batch processes," Canadian Journal of Chemical Engineering, vol. 96, no. 2, pp. 521-530, 2018.

[28] H. Tao, W. Paszke, E. Rogers, H. Yang, and K. Gałkowski, "Iterative learning fault-tolerant control for differential timedelay batch processes in finite frequency domains," Journal of Process Control, vol. 56, pp. 112-128, 2017.

[29] H. Tao, Y. Liu, and H. Yang, "Output information based fault-tolerant iterative learning control for dual-rate sampling process with disturbances and output delay," Mathematical Problems in Engineering, vol. 2018, 15 pages, 2018. 
[30] L. Feng, K. Zhang, Y. Chai, S. Xu, and Z. Yang, "Iterative learning fault estimation design for nonlinear system with random trial length," Complexity, vol. 2017, Article ID 1850737, 9 pages, 2017.

[31] L. Feng, Y. Chai, S. Xu, and Z. Yang, "Observer-based fault estimators using iterative learning scheme for nonlinear time-delay systems with intermittent faults," International Journal of Robust and Nonlinear Control, vol. 27, no. 17, pp. 1-9, 2017.

[32] L. Feng, S. Xu, Y. Chai, Z. Yang, and K. Zhang, "Iterative learning scheme to design intermittent fault estimators for nonlinear systems with parameter uncertainties and measurement noise," International Journal of Adaptive Control and Signal Processing, vol. 32, no. 7, pp. 994-1009, 2018.

[33] B. Shen, S. X. Ding, and Z. Wang, "Finite-horizon $H_{\infty}$ fault estimation for uncertain linear discrete time-varying systems with known inputs," IEEE Transactions on Circuits and Systems II Express Briefs, vol. 60, no. 12, pp. 902-906, 2013.

[34] M. M. Seron and J. A. de Doná, "Robust fault estimation and compensation for LPV systems under actuator and sensor faults," Automatica, vol. 52, pp. 294-301, 2015.

[35] E. Tian, D. Yue, and G. Wei, "Robust $\mathrm{H}_{\infty}$ filter for discretetime linear system with uncertain missing measurements and non-linearity," IET Signal Processing, vol. 7, no. 3, pp. 239248, 2013.

[36] J. Hu, D. Chen, and J. Du, "State estimation for a class of discrete nonlinear systems with randomly occurring uncertainties and distributed sensor delays," International Journal of General Systems, vol. 43, no. 3-4, pp. 387-401, 2014.

[37] H. Dong, Z. Wang, S. X. Ding, and H. Gao, "Finite-horizon reliable control with randomly occurring uncertainties and nonlinearities subject to output quantization," Automatica, vol. 52, pp. 355-362, 2015.

[38] W. Chen, A. Khan, M. Abid, and S. Ding, "Integrated design of observer based fault detection for a class of uncertain nonlinear systems," International Journal of Applied Mathematics and Computer Science, vol. 21, no. 3, pp. 423-430, 2011.

[39] Z. Ke, B. Jiang, S. Peng, and J. Xu, "Analysis and design of robust $H_{\infty}$ fault estimation observer with finite-frequency specifications for discrete-time fuzzy systems," IEEE Transactions on Cybernetics, vol. 45, no. 7, pp. 1225-1235, 2015.

[40] Y. Jia, "Alternative proofs for improved LMI representations for the analysis and the design of continuous-time systems with polytopic type uncertainty: a predictive approach," IEEE Transactions on Automatic Control, vol. 48, no. 8, pp. 14131416, 2003. 


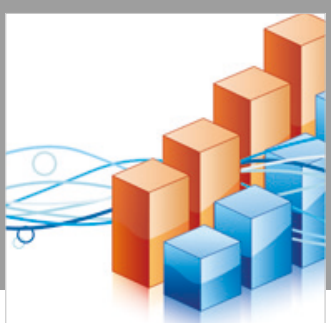

Advances in

Operations Research

\section{-n-m}
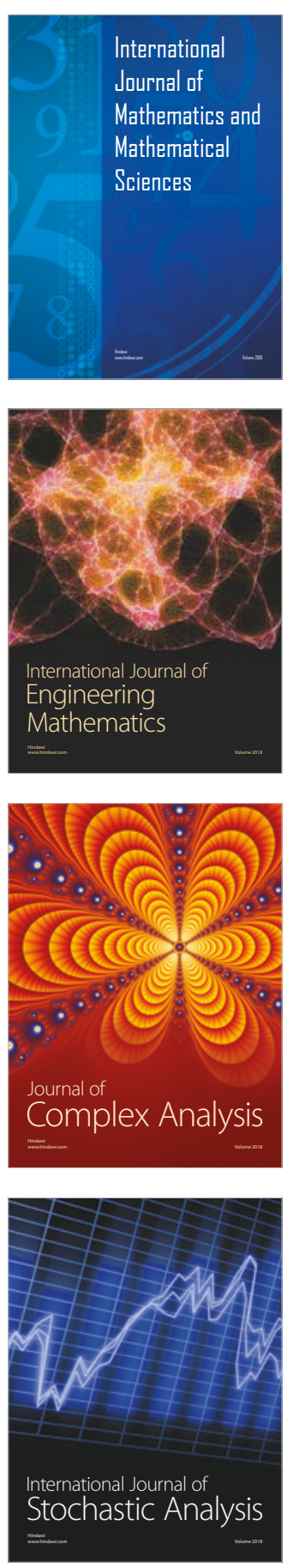
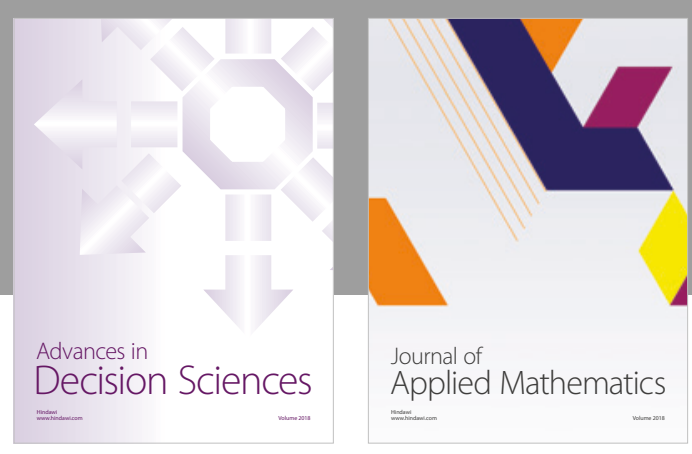

Journal of

Applied Mathematics
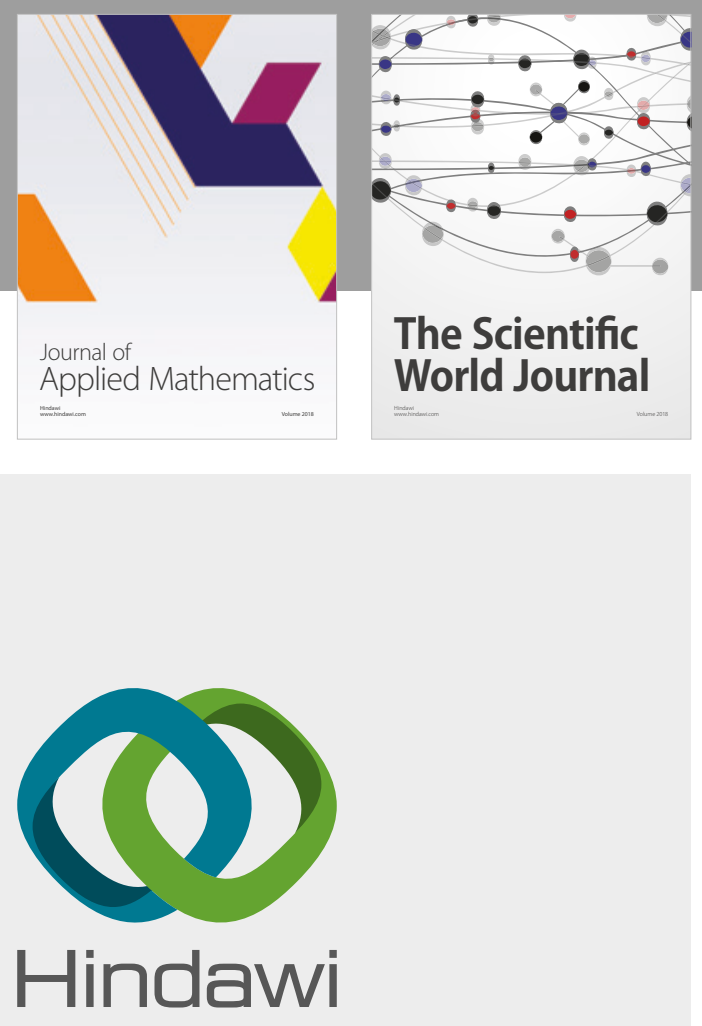

Submit your manuscripts at

www.hindawi.com

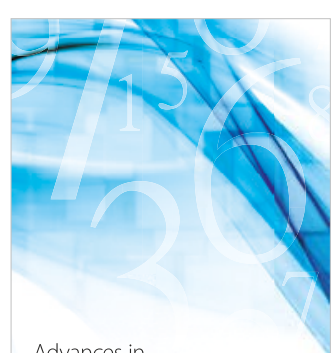

Advances in
Numerical Analysis
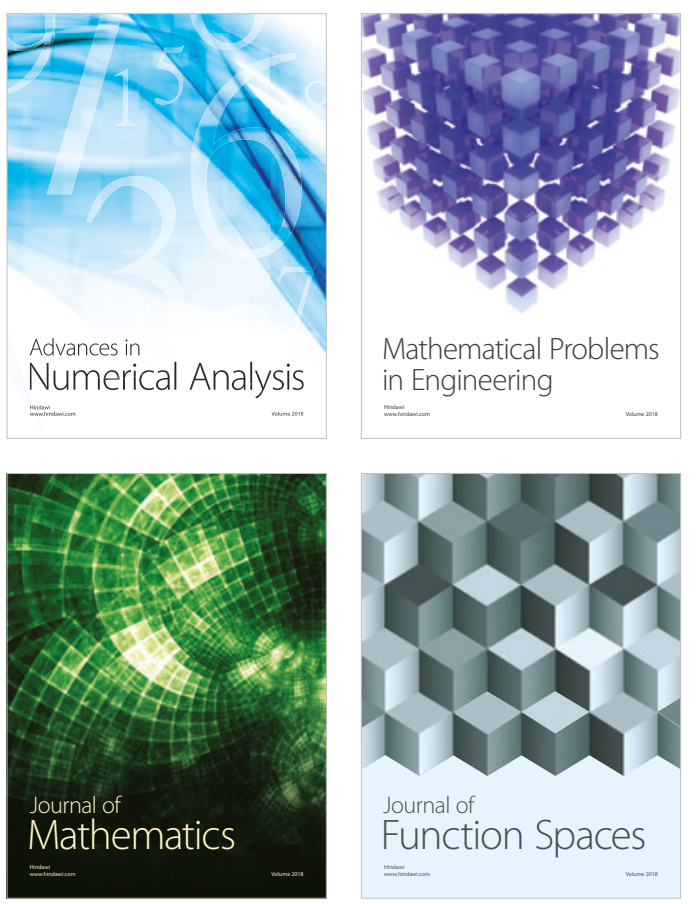

Mathematical Problems in Engineering

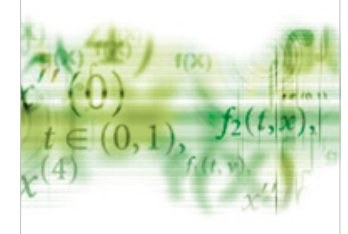

International Journal of

Differential Equations

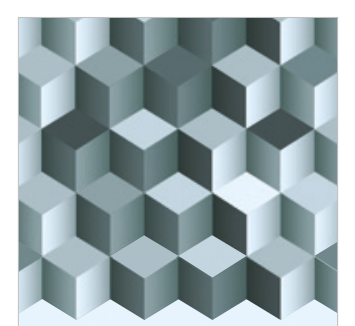

Journal of

Function Spaces
The Scientific

World Journal

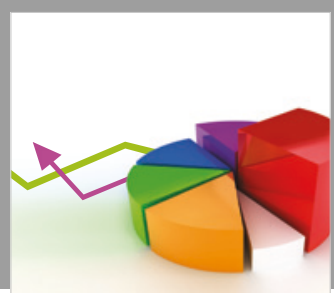

Journal of

Probability and Statistics
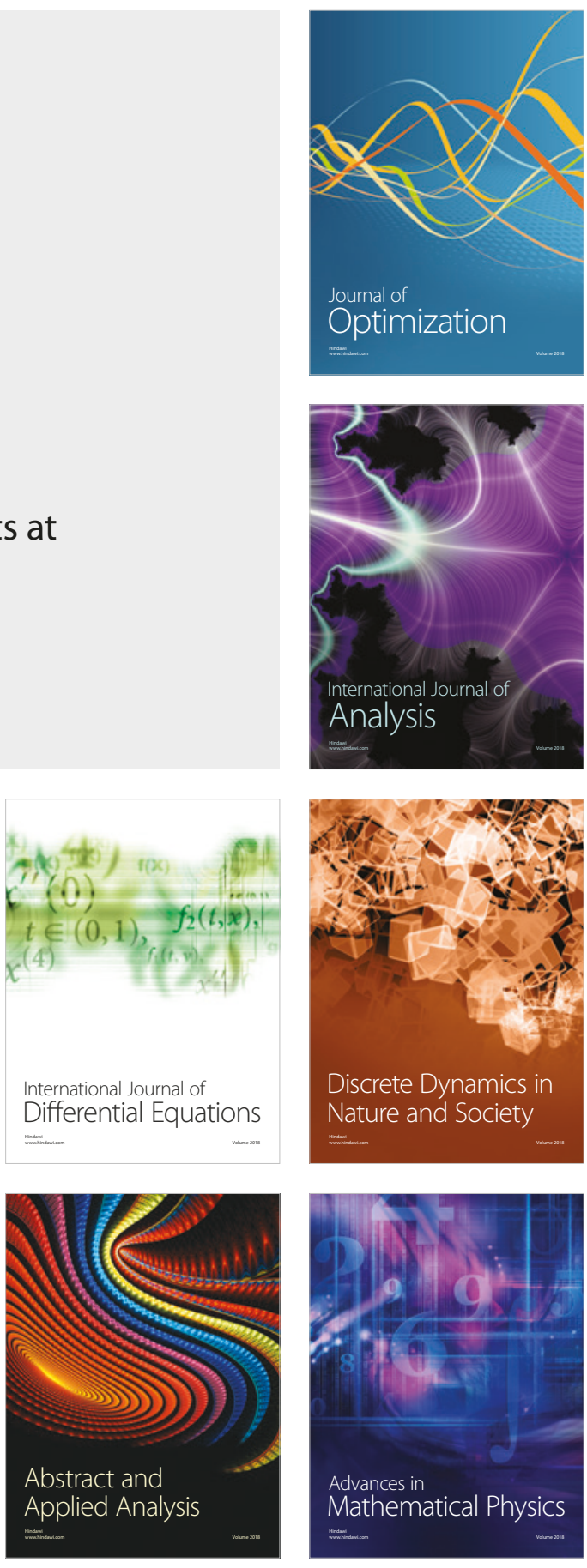